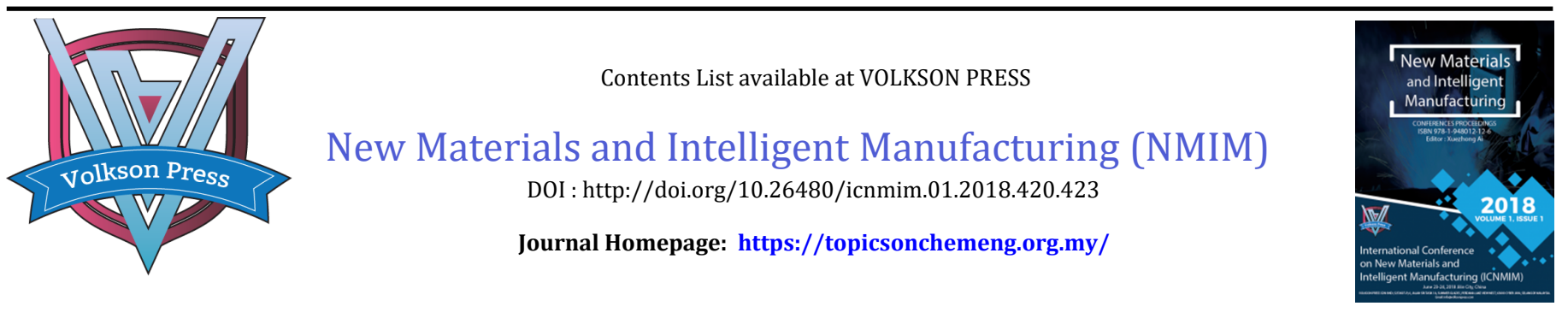

ISBN: 978-1-948012-12-6

\title{
DESIGN AND CALCULATION OF PIEZOELECTRIC POWER GENERATION DEVICE ON TIRE PRESSURE MONITORING SYSTEM
}

\author{
Jie Meng1 ${ }^{*}$, Sun Xiaofeng1, Jiang Delong ${ }^{2}$, Zhang Guangchao ${ }^{3}$ \\ ${ }^{1}$ College of mechanical and electrical engineering, Jilin Institute of Chemical Technology, Jilin,China \\ ${ }^{2}$ Department of assets, Jilin Institute of Chemical Technology, Jilin,China \\ ${ }^{3}$ The 3rd Institute, China Aerospace Science and Industry Corporation Limited \\ *Corresponding Author E-mail: jiemeng1980@126.com
} This is an open access article distributed uder the Creative Commons Attribution License, which permits unrestricted use, distribution, and reproduction in any
medium, provided the original work is properly cited

\section{ARTICLE DETAILS}

Article History:

Received 26 June 2018

Accepted 2 July 2018

Available online 1 August 2018

\section{ABSTRACT}

The structure of piezoelectric power generation device on tire pressure monitoring system was put forward in this paper. The formula for the energy output of the piezoelectric generator was solved. The natural frequency of the power generation device and the frequency of the external excitation can be matched by adjusting the quality of the mass block to improve the power generation capability.

\section{KEYWORDS}

Piezoelectric power generation device, Tire pressure monitoring system, piezoelectric vibrator.

\section{INTRODUCTION}

TPMS (Tire Pressure Monitoring System) is used for monitoring the pressure and temperature of the tire when the car is moving. TPMS, as a active safety warning system, will alarm the driver in order to ensure the traffic safety when the pressure is not enough or leaked the temperature is too high or low, because it can monitor the condition of the tire in realtime. The TPMS is battery-powered by conventional way, but it needs replace battery regularly because of its limited life. To solve this problem, we expanded the research on through the piezoelectric to power the detection and transmitter module in the paper. Based on a study, Piezoelectric vibrator is the important part of the piezoelectric power generator which directly affects the performance of the piezoelectric power generator. So, we need to research its performance parameters [14].

Piezoelectric power generation device which is use of piezoelectric vibrator to harvest mechanical force to generate electrical energy is called micro-electricity generation device that is an important part and researching focus in micro-power generation. There are more than ten counties and regions all over the world in which there are the research institutions of piezoelectric power generation. With many merits such as simple structure, small body, no interfere of electromagnetism and easy to implement, it has broad application prospects in low-power radio frequency field.

\section{STRUCTURE COMPOSITION OF PIEZOELECTRIC POWER GENERATION DEVICE}

When the cantilever beam piezoelectric vibrator is selected for power generation, more electric energy can be obtained. It can be known from theoretical and experimental analysis that when the piezoelectric vibrator is used to generate power, only when the external excitation frequency is close to its natural frequency, the power generation capacity and energy conversion efficiency are higher. We could know that the natural frequency of the piezoelectric vibrator is about $180 \mathrm{~Hz}$ by the test, and with an external excitation on frequency lower than $90 \mathrm{~Hz}$, the performance of power generation will be greatly reduced. In order to make the power generation performance of the piezoelectric power generation device to be maximized, and masses are often provided at the ends of the piezoelectric vibrators to adjust the natural frequency of the system, and make sure that the power generation capability of the power generation device is in an optimal state.

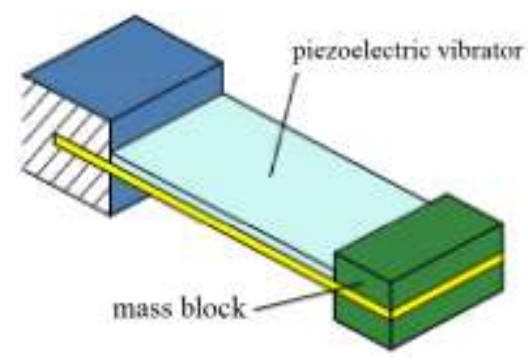

Figure 1: Cantilever beam piezoelectric power generation device

The structure of a micro-piezoelectric power generation device (hereinafter referred to as a cantilever beam piezoelectric power generation device or a piezoelectric power generation device) is composed of a support shell, a piezoelectric vibrator, and a mass block and the voltage output. (e.g. Figure 1). The specific working process is described as follows: In the illustrated structure, when the free end or the supporting end of the piezoelectric vibrator is excited, it will generate a bending deformation, thereby generating a charge on the surface of the plate. The external performance of the piezoelectric oscillator's output energy is voltage.

\section{CALCULATION OF ENERGY OUTPUT OF PIEZOELECTRIC POWER GENERATION DEVICE}

According to the elastic theory of beams, the displacement at any point in the direction $x$ of the beam can be expressed as follows $[5,6]$

$$
w_{x}=w_{0}(x, t)-y_{p} \frac{\partial \mu(x, t)}{\partial t}
$$


Among them, $w_{0}$ is the displacement of the shaft, $y_{p}$ is the distance between the center axis of the piezoelectric substrate and the neutral axis of the piezoelectric vibrator, $\mu$ is the longitudinal displacement of the beam. The axial strain of the beam can be expressed as:

$$
\varepsilon_{1}=\frac{\partial w_{x}}{\partial x}=-\frac{\partial^{2} u}{\partial x^{2}} y_{p}
$$

According to the basic theory of piezoelectric ceramics, the electric displacement of the piezoelectric substrate along the axis $z$ of the piezoelectric vibrator can be expressed by the relationship between the strain in the direction of the piezoelectric substrate and the electric field, as follows:

$$
D_{31}=e_{31} \varepsilon_{1}+\varepsilon_{33}^{T} E
$$

Since the applied electric field is 0 , the electric displacement of the piezoelectric substrate can be expressed by the following equation:

$$
D_{31}=e_{31} \varepsilon_{1}=d_{31} E_{p} \varepsilon_{1}
$$

Therefore, the total amount of charge generated by piezoelectric vibrators is:

$$
\begin{aligned}
Q_{u n} & =\int_{A} D_{31} d A \\
& =W d_{31} E_{p} y_{p, u n} \int_{0}^{L}-\frac{\partial^{2} u}{\partial x^{2}} d x \\
& =\frac{429 W d_{31} E_{p} y_{p, u n} \sqrt{0.243 W L\left(\rho_{m} h_{m}+\rho_{p} h_{p}\right)} \omega^{2} y_{0}}{200 L\left[\left(\omega_{i, u n}^{2}-\omega^{2}\right)^{2}+\left(2 \zeta_{u n} \omega_{i, u n} \omega\right)^{2}\right] 1 / 2} \cos (w t-\varphi) \\
Q_{b i} & =2 \int_{A} D_{31} d A \\
& =2 W d_{31} E_{p} y_{p, b i} \int_{0}^{L}-\frac{\partial^{2} u}{\partial x^{2}} d x \\
& =\frac{429 W d_{31} E_{p} y_{p, b i} \sqrt{0.243 W L\left(\rho_{m} h_{m}+2 \rho_{p} h_{p}\right)} \omega^{2} y_{0}}{100 L\left[\left(\omega_{i, b i}^{2}-\omega^{2}\right)^{2}+\left(2 \zeta_{b i} \omega_{i, b i} \omega\right)^{2}\right] 1 / 2} \cos (w t-\varphi)
\end{aligned}
$$

When the piezoelectric vibrator operates at a resonant frequency ( $\omega=\omega_{i}$ ), it generates the largest amount of power.

$$
\begin{aligned}
& Q_{\text {max }, \text { un }}=\int_{A} D_{31} d A \\
& =W d_{31} E_{p} y_{p, \text { un }} \int_{0}^{L}-\frac{\partial^{2} u}{\partial x^{2}} d x \\
& =\frac{429 W d_{31} E_{p} y_{p, \text { un }} \sqrt{0.243 W L\left(\rho_{m} h_{m}+\rho_{p} h_{p}\right)} y_{0}}{200 L \zeta_{u n}} \\
& Q_{\max , b i}=2 \int_{A} D_{31} d A \\
& =2 W d_{31} E_{p} y_{p, b i} \int_{0}^{L}-\frac{\partial^{2} u}{\partial x^{2}} d x \\
& =\frac{429 W d_{31} E_{p} y_{p, b i} \sqrt{0.243 W L\left(\rho_{m} h_{m}+2 \rho_{p} h_{p}\right)} y_{0}}{100 L \zeta_{b i}}
\end{aligned}
$$

From the Gaussian formula $W=\frac{Q^{2}}{2 C}$, it can be seen that the electrical energy generated by the piezoelectric vibrators is:

$$
\begin{aligned}
W_{\text {max }, u n}= & \frac{Q_{\text {max }, u n}^{2}}{2 C_{u n}}=\left[\frac{429 W d_{31} E_{p} y_{p, u n} \sqrt{0.243 W L\left(\rho_{m} h_{m}+\rho_{p} h_{p}\right)} y_{0}}{200 L \zeta_{u n}}\right]^{2} / \frac{2 \varepsilon_{33}^{T} W L}{(1-\alpha) h} \\
& =\frac{7 d_{31}^{2} E_{p}^{2} y_{0}^{2} W^{2} h^{4} \alpha^{2} \gamma^{2}\left[\rho_{m} \alpha+\rho_{p}(1-\alpha)\right](1-\alpha)}{50 \varepsilon_{33}^{T} \zeta_{u n}^{2} L^{2}(1-\alpha+\alpha \gamma)^{2}}
\end{aligned}
$$

$$
\begin{aligned}
W_{\text {max }, b i}= & \frac{Q_{\text {max }, b i}^{2}}{2 C_{b i}}=\left[\frac{429 W d_{31} E_{p} y_{p, b i} \sqrt{0.243 W L\left(\rho_{m} h_{m}+2 \rho_{p} h_{p}\right)} y_{0}}{100 L \zeta_{b i}}\right]^{2} / \frac{8 \varepsilon_{33}^{T} W L}{(1-\alpha) h} \\
& =\frac{7 d_{31}^{2} E_{p}^{2} y_{0}^{2} h^{4} W^{2}\left[\rho_{m} \alpha+\rho_{p}(1-\alpha)\right](1-\alpha)(1+\alpha)^{2}}{200 L^{2} \varepsilon_{33}^{T} \zeta_{b i}^{2}}
\end{aligned}
$$

From the above formula, it can be known that the piezoelectric vibrator could generates electricity by vibration. The energy generated by the piezoelectric vibrator is proportional to the square of the ratio of width to length and is also proportional to the fourth power of the thickness. The related parameters of the structure and material also have a great influence on the energy output.

\section{EXPERIMENTS OF THE PIEZOELECTRIC POWER GENERATION DEVICE}

The structural dimensions of the piezoelectric vibrator, the weight of the end mass, and the external excitation all have a great influence on its power generation capability. When the structure size of the piezoelectric generating device is constant, there exists an optimum excitation frequency to maximize its power generation capacity. This is because when the external excitation frequency is close to its natural frequency, the piezoelectric vibrator has a higher power generation capacity. The resonant frequency of the entire device can be reduced by changing the quality of the mass block. It can be seen from Figure 2 by experiments that the piezoelectric power generation device increases with the quality of the mass block. While the power generation capacity is increasing, the best frequency it needs to reach the maximum is gradually decreasing. When the power is generated by the piezoelectric power generation device, the natural frequency of the power generation device and the frequency of the external excitation can be matched by adjusting the quality of the mass to improve the power generation capability.

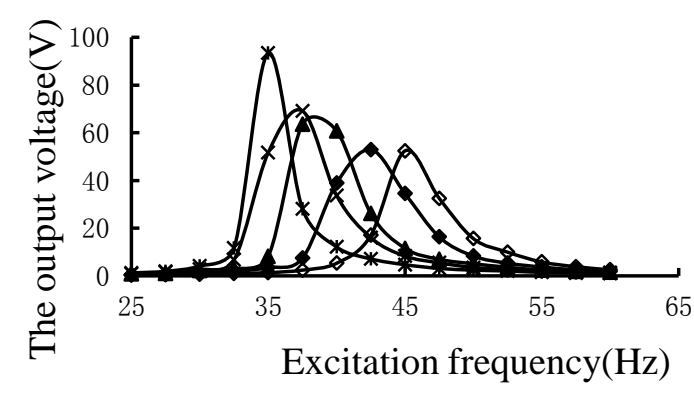

Figure 2: Relationship between output voltage and excitation frequency

\section{CONCLUSIONS}

The structure of piezoelectric power generation device on tire pressure monitoring system was put forward in this paper, and it was composed of a support shell, a piezoelectric vibrator, and a mass block. The formula for the energy output of the piezoelectric generator was solved. The natural frequency of the power generation device and the frequency of the external excitation can be matched by adjusting the quality of the mass to improve the power generation capability.

\section{REFERENCES}

[1] Allameh, S.M., Akogwu, O., Collinson, M. 2007. Piezoelectric generators for biomedical and dental applications: Effects of cyclic loading Journal of Materials Science: Materials in medicine, 18, 39-45.

[2] Lai, E., Redfern, A., Wright, P. 2005. Vibration Powered BatteryAssisted Passive RFID Tag. Japan, Nagasaki-shi.

[3] Lewandowski, B.E., Kilgore, K.L., Gustafson, K.J. 2007. Design Considerations for an Implantable, Muscle Powered Piezoelectric System for Generating Electrical Power. Journal of Annals of Biomedical Engineering, 35 (4).

[4] Ferrari, M., Ferrari, V., Guizzetti, M., Marioli, D., Taroni, A. 2007. Piezoelectric Multifrequency Energy Converter for Power Harvesting in Autonomous Microsystems. Journal of Sensors and Actuators, 142, 329335. 
[5] Liu, Q.Q. 1998. Theoretical mechanics. Changchun, Jilin Science and Technology Press, 212-226.
[6] Zhang, G.C. 2011. Research on design theory and experiment of piezoelectric power generation device on tire pressure monitoring system, Jilin University. 